\title{
Retrospective Review of Rapid Pediatric Brain MR Imaging at an Academic Institution Including Practice Trends and Factors Affecting Scan Times
}

\author{
B.D. Niederhauser, R.J. McDonald, L.J. Eckel, G.F. Keating, E.M. Broomall, N.M. Wetjen, F.E. Diehn, K.M. Schwartz, C.H. Hunt,
}

K.M. Welker, and D.F. Kallmes

\begin{abstract}
BACKGROUND AND PURPOSE: In an effort to reduce radiation exposure in children requiring regular follow up for shunted hydrocephalus, our institution implemented a rapid brain MR imaging protocol. The purpose of this study was to review an academic practice experience with pediatric rapid brain MR imaging without patient sedation in the evaluation of hydrocephalus and a limited group of other conditions.
\end{abstract}

MATERIALS AND METHODS: We retrospectively analyzed limited-sequence, rapid brain MR imaging scans performed in nonsedated patients younger than 14 years between April 2009 and December 2011. So-called failed examinations were determined by consensus of 2 authors as insufficiently diagnostic for evaluation of ventricular size. CT and MR imaging quarterly volumes for hydrocephalus-related indications were determined from 2005-2012. Multivariable logistic regression analysis was performed to elucidate factors potentially affecting scan durations including examination indication and patient age, sex, inpatient status, and clinical conditions.

RESULTS: A total of 398 examinations were performed on 168 patients (103 boys, 65 girls; median age, 13 months). None were deemed to be failed examinations. Median scan duration was 4.43 minutes (interquartile range, 4.42 minutes-5.88 minutes; SD, 2.42 minutes). Examination indication of altered mental status was the only factor associated with increased scan duration $(+1.77$ minutes; $P=.0021)$. Hydrocephalus-related imaging volumes approximately doubled in the 7 years reviewed, but rapid MR imaging introduced in 2009 is quickly replacing CT scanning for these indications, accounting for nearly 7 of every 8 examinations at the end of the study period.

CONCLUSIONS: In every case of initial work-up and follow-up, rapid brain MR imaging effectively evaluated ventricular size and/or intracranial fluid and represents a viable alternative to CT scanning, irrespective of a child's age or clinical condition. For this indication and patient group, MR imaging is now the predominant imaging method in our practice.

ABBREVIATIONS: EMR = electronic medical record; HASTE = half-Fourier acquisition single-shot turbo spin-echo; true FISP = true fast imaging with steady-state precession

$\mathrm{C}^{1}$

scanning has historically been the preferred imaging technique for the initial evaluation and follow-up of hydrocephalus and intracranial cysts within the pediatric population at many institutions. Compared with traditional MR imaging methods, pediatric head CT imaging offers much faster acquisition times, comparable anatomic resolution, and lower patient cost.

Received November 21, 2012; accepted after revision December 12.

From the Department of Radiology (B.D.N., R.J.M., L.J.E., F.E.D., K.M.S., C.H.H., K.M.W., D.F.K.), Clinician Investigator Training Program (R.J.M.), and Departments of Neurology (G.F.K., E.M.B.) and Neurosurgery (N.M.W., D.F.K.), Mayo Clinic, Rochester, Minnesota.

Paper previously presented at: Annual Meeting of the American Society of Neuroradiology, April 22, 2012; New York, New York.

Please send correspondence to Laurence J. Eckel, MD, Department of Radiology, Mayo Clinic, 200 1st St. SW, Rochester, MN 55905; e-mail:

eckel.laurence@mayo.edu

三 Indicates article with supplemental on-line tables.

http://dx.doi.org/10.3174/ajnr.A3510
Furthermore, the prolonged scan time of traditional MR imaging methods often demands use of general anesthesia in pediatric patients because of poor compliance and image motion artifacts. Such measures significantly increase cost and result in risk from anesthetic administration ${ }^{1-3}$ and result in substantial delays within the imaging suite. However, ongoing parental and physician concern for the potential long-term effects of ionizing radiation, ${ }^{4-6}$ particularly to young patients who require subsequent imaging studies, have resulted in increased interest in alternatives to CT scanning. The Alliance for Radiation Safety in Pediatric Imaging, a collaboration of 13 leading medical societies, has led the way in promoting appropriate low-dose and limited use of radiation in pediatric imaging through its Image Gently campaign, ${ }^{7}$ with an emphasis of the Society for Pediatric Radiology As Low as Reasonably Achievable (ALARA) principles. ${ }^{8}$

Recent developments in MR pulse sequences now allow for rapid evaluation of ventricular size in the pediatric population 
without the need for sedation. ${ }^{9-14}$ Although various techniques have been used, heavily T2-weighted single-shot fast spin-echo and gradient recalled-echo imaging are the 2 most common pulse sequence methods used to interrogate ventricular size and shunt catheter tip location, respectively. ${ }^{15}$ Starting in April 2009, our institution introduced a similar T2-weighted HASTE rapid-sequence MR imaging protocol.

A significant factor affecting resolution of anatomic detail in MR imaging is patient motion, which is directly related to the time required to complete each sequence. Thus, shorter sequences are desirable and beneficial. Heavily T2-weighted sequences, which typically require less than 30 seconds of imaging for each anatomic plane, provide excellent imaging with high intrinsic contrast between intracranial fluid and brain tissue and maintain short scan durations for the nonsedated child. Standard spin-echo sequences can require up to several minutes to complete, which is an unacceptably long time for a nonsedated and potentially uncooperative infant or child. Our institution, along with most radiology practices, is continually striving to minimize scan durations, not only for the purpose of patient comfort but also for efficient patient throughput. Understanding patient factors that increase scan durations could potentially guide informed consent discussions before examinations, as well as triage potential socalled failed examinations that may require sedation or general anesthesia for diagnostic MR imaging. The purpose of this study was to review an academic practice experience with pediatric rapid brain MR imaging without the need for patient sedation in the evaluation of hydrocephalus and a limited group of other conditions.

\section{MATERIALS AND METHODS Study Design and Data Extraction}

Design and implementation of this single-center retrospective study met institutional review board and Health Insurance Portability and Accountability Act guidelines. Informed consent was waived. All data for this study were extracted from the institutional EMR. Selected imaging and clinical notes within the EMR were searched to identify clinical indications and comorbidities pertinent to this study. Scanner time and total imaging time were extracted from the proprietary institutional radiology imageviewing platform. The total number of pediatric imaging examinations, sorted by month, was extracted from the radiology information management system.

For each patient record identified, the following data were extracted from the EMR: date of scan, scan type and duration, magnet strength of the scanner, primary diagnoses, imaging indications, whether a ventricular shunt was in place, patient location (inpatient, outpatient, or emergency department), and demographic data including date of birth and sex. The number and type of each scan sequence, including repeated scans and plane of imaging, were also recorded. Total scan duration was determined from differences in image time stamps between the first scout image and the last sequences obtained.

Departmental imaging volumes for hydrocephalus, including the number of rapid MR imaging and pediatric head CT scans performed per month, were collected. The number of noncontrast CT examinations during the 3 -year period leading up to the introduction of the rapid MR imaging protocol was determined by searching the radiology data base for all pediatric head CT examinations with indications of "hydrocephalus," "shunt," "cyst," "macrocephaly," "ventricles," "ventricular," "aqueductal stenosis," and "vp" and was compared with rapid MR imaging examinations performed for the same indications.

\section{Imaging Protocol}

Our rapid MR imaging technique used for evaluation of ventricular size in pediatric patients was HASTE, a T2-weighted MR imaging technique (matrix size, $256 \times 179$; field of view, $22 \mathrm{~cm}$; TR, 2000 ms; TE, 105 ms; section thickness, 4 mm; imaging time to cover the entire brain, approximately 30 seconds per plane, typically in both axial and coronal planes). This technique was implemented at our institution beginning in April 2009. Additional imaging sequences were added ad hoc as appropriate per the interpreting radiologist's discretion to avoid repeated or return examinations. In this patient population, additional sequences most commonly consisted of sagittal views or multiplanar T2 balanced gradient true FISP sequences (matrix size, $256 \times$ 192; field of view, $29 \mathrm{~cm}$; TR, $3.3 \mathrm{~ms}$; TE, $1.3 \mathrm{~ms}$; flip angle, 70 degrees; section thickness, $4 \mathrm{~mm}$ ).

\section{Patient Population}

Inclusion criteria were patients younger than 14 years with suspected or confirmed hydrocephalus or intracranial cysts who underwent a rapid MR imaging scan between April 1, 2009, and December 31, 2011. Patients were excluded if the limited MR imaging study was not performed according to defined rapidsequence protocol parameters. In addition, imaging studies that included 3 or more pulse-sequence types or administration of intravenous contrast were excluded because they did not meet the strict definitions of a rapid MR imaging protocol.

\section{Definition of Failure}

Rapid MR imaging failure was defined as an examination unable to answer the clinical question (most commonly, ventricular size assessment) because of inadequate resolution or enough patient motion artifacts requiring further imaging with same-day or next-day subsequent rapid MR imaging, CT imaging, or MR imaging with the patient under sedation. To determine whether a scan was considered a failure, we searched radiology reports for key words or phrases such as inadequate and nondiagnostic or whether recommendation was made to pursue additional imaging with CT scanning, repeated MR imaging, or MR imaging with the patient under sedation. In addition, we reviewed radiology reports for all head CT and MR imaging examinations performed within 2 weeks subsequent to the identified rapid MR imaging examination, along with the accompanying clinical notes to further gain an understanding of whether multiple examinations in a short period were the result of rapid MR imaging failure. Cases of potential failure were analyzed by a consensus of 2 radiologists (B.D.N. and L.J.E.) after full clinical and radiologic record review. Cases requiring additional imaging to further evaluate findings other than the ventricle size or shunt position on the limited MR imaging, such as mass, encephalocele, etc, were not considered failures of rapid MR imaging.

AJNR Am J Neuroradiol 34:1836-40 Sep 2013 www.ajnr.org 


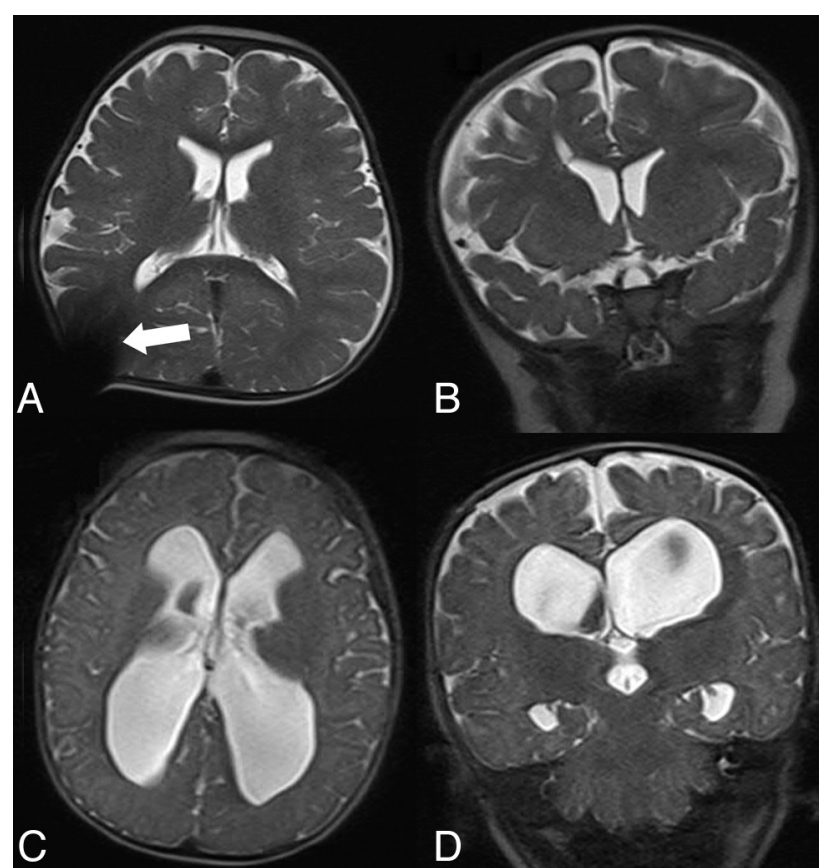

FIG 1. Axial $(A)$ and coronal $(B)$ HASTE sequence MR imaging of the brain in a 4-year-old child with shunted hydrocephalus demonstrating excellent delineation of the ventricles and mild shunt reservoir artifacts (white arrow) with few motion artifacts despite lack of sedation. Similar axial $(C)$ and coronal $(D)$ HASTE MR imaging of the brain in a 6-month-old child performed for follow-up of known hydrocephalus demonstrating adequate ventricular delineation despite significant patient motion.

\section{Statistical Methods}

We performed all statistical analyses by using JMP version 9 (SAS Institute, Cary, North Carolina). Categoric data were displayed as relative frequencies (percentages) and were compared by use of $\chi^{2}$ tests. Continuous data were presented as median scores with interquartile ranges because of non-normal data distributions, and were compared by used of the Wilcoxon signed-rank test of significance. Linear regression models were generated to determine the effect of imaging indication, demographic variables (age, sex), and comorbidities on total scan and examination room times. We estimated trends in imaging volumes with time by using regression analysis.

\section{RESULTS}

\section{Demographic and Clinical Characteristics}

A total of 404 rapid MR imaging examinations were performed in 168 patients during the designated time. Six examinations were excluded for ad hoc addition of at least $3 \mathrm{MR}$ imaging sequences, for a total study sample of 398 rapid MR imaging examinations. Examinations were performed on various MR imaging scanners, including 1.5 and 3T; however, 341 (86\%) of 398 examinations were completed on a Magnetom Espree 1.5T MR imaging scanner with a 70-cm bore (Siemens, Erlangen, Germany). The demographic characteristics of the study population are shown in On-line Table 1. All examinations consisted of HASTE images in the axial plane, and nearly all (>99\% [397/398]) examinations included additional coronal plane acquisitions (Fig 1). A total of 265 (67\%) of 398 examinations were performed on patients in the outpatient setting, 93 (23\%) on inpatients, and $40(10 \%)$ on patients in the emergency department. Of these 398 examinations, 248 (62\%) were performed on patients with ventricular shunts in place.

\section{Study Indications}

The breakdown of indications and clinical situations for all rapid MR imaging examinations is shown in On-line Table 1. Evaluation of ventricular size was the most common reason for ordering rapid MR imaging in this population, accounting for 318 (79.9\%) of 398 imaging indications. Less common requests included follow-up of known intracranial blood or fluid (9.0\% [36/398]), unspecified macrocephaly (3.0\% [12/398]), and evaluation of intracranial cyst (2.3\% [9/398]). A total of 23 (5.8\%) of 398 requests were performed for other reasons, including evaluation of known encephalocele, infarction, or intracranial mass. Of the 327 examinations performed specifically to evaluate ventricular size or intracranial cyst, $162(49.5 \%)$ were performed as routine clinical follow-up in asymptomatic patients; 38 (11.6\%) for headache; 37 (11.3\%) for postoperative evaluation; 35 (10.7\%) for vomiting; 23 (7.0\%) for "shunt malfunction"; 19 (5.8\%) for altered mental status; 12 (3.7\%) for macrocephaly; 6 (1.8\%) for "fussy"; 5 (1.5\%) for seizure; and $15(4.6 \%)$ for other clinical symptoms such as localized pain, history of intracranial hemorrhage, or cranial dysmorphism. Some patients $(21 / 327,6.4 \%)$ had multiple symptoms such as headache plus vomiting.

\section{Rapid MR Imaging Study Quality and Failure}

No examination failures were identified. That is, all (398/398) rapid MR imaging examinations in this study provided the diagnostic information necessary to answer the clinical question posed. This included 9 (2.3\%) of 398 rapid MR imaging reports that described image degradation by motion artifacts. A total of 6 $(1.5 \%)$ same-day or next-day examinations were performed with either MR imaging with the patient under sedation $(n=2)$ or with CT imaging $(n=4)$. However, none of these were determined to have been the result of inadequate rapid MR imaging, and each was performed to obtain additional data not available on rapid MR imaging. These cases included: 1 ) a patient who had a seizure in the MR imaging suite after images were obtained and was then transferred to CT for an emergent scan; 2) a patient who presented to the emergency department with symptoms of obstructive hydrocephalus on 2 consecutive weeks and had negative results on rapid MR images, but then was referred within 48 hours for CT scan on the next similar presentation, which was also negative for hydrocephalus; 3 ) a patient who was evaluated from the emergency department with MR imaging for symptoms of obstructive hydrocephalus who received a CT for evaluation of possible craniosynostosis the following day; 4) a patient who had a report of a mass on an outside CT scan and received a rapid MR imaging for confirmation and an operative planning CT scan the following day; 5) a patient in whom an unusual intracranial mass abutting the inner table of the skull was identified by rapid MR imaging and a same-day CT scan was obtained to evaluate further for bony erosion; and 6) a patient with craniosynostosis, ventriculoperitoneal shunt, and an encephalocele being evaluated for shunt malfunction who received a next-day CT scan because the radiology report stated that the encephalocele was not well seen. 


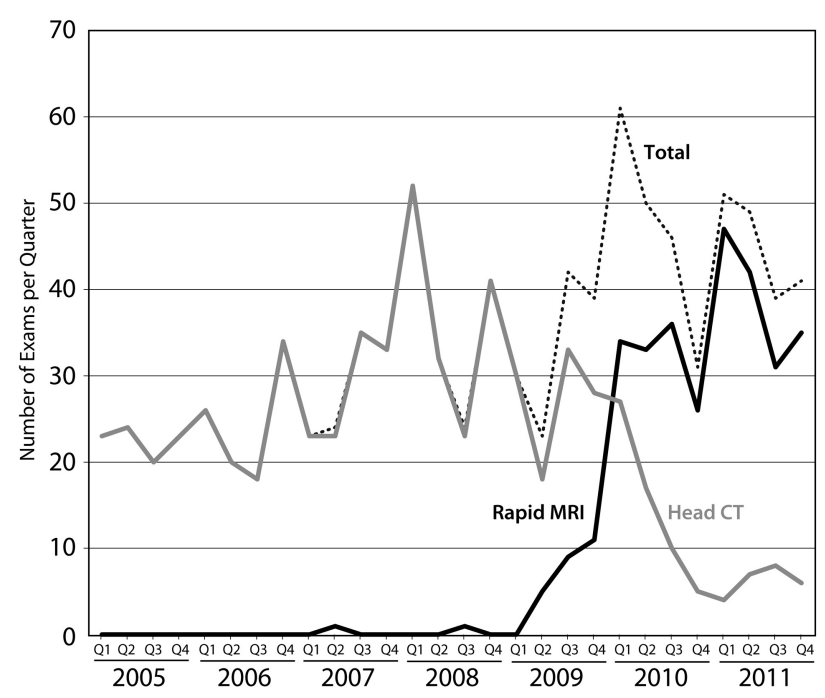

FIG 2. Trends in CT and rapid MR imaging use for hydrocephalusrelated conditions, 2005-2011. Note that although overall imaging volumes for these indications have increased, rapid MR imaging is rapidly replacing $\mathrm{CT}$ imaging.

None of these 6 cases overlapped with those 9 in which reports mentioned significant motion.

\section{Rapid MR Imaging Performance}

The average scan duration was 4.43 minutes (SD, 2.42 minutes). Linear regression analysis (On-line Table 2) revealed that altered mental status, as an indication for imaging, was the only variable significantly associated with longer scan duration, resulting in increased average scan duration of 6.20 minutes compared with 4.43 minutes (On-line Table 2). No other indication, comorbidity, or demographic variable was significantly associated with scan duration. There were $40(10.1 \%)$ of 398 examinations that had additional sequences added on ad hoc by the interpreting radiologist, most commonly axial gradient-echo true FISP sequences.

\section{Trends in Rapid MR Imaging Use}

During the 6-year period of this study, 643 unenhanced CT scans were performed in pediatric patients younger than 14 years for indications relating to intracranial cyst or ventricular size. From the beginning of 2005 to the end of 2011, the average number of monthly imaging studies used to diagnose ventricular pathologic conditions has increased from an average of 22.5 to 45 (Fig 2). Before mid-2009, this upward trend was entirely attributable to greater use of pediatric CT scanning. However, beginning in the second quarter of 2009, rapid MR imaging has steadily replaced CT scanning as the primary technique used for evaluation of ventricular pathologic conditions in the pediatric population. In the last quarter of 2011, rapid MR imaging accounted for 85.4\% (35/ 41) of all cross-sectional brain imaging performed on children with suspected or known ventricular abnormalities, not including transcranial sonography.

\section{DISCUSSION}

The results from our retrospective study indicate that, across a wide age range, rapid-scan pediatric brain MR imaging with the patient nonsedated allows fast and successful assessment of ven- tricular size without the need for repeated examination because of motion artifacts or poor study quality. Referral patterns within our institution suggest that providers are increasingly favoring rapid MR imaging vs CT scanning for pediatric ventricular evaluation. In addition, our study demonstrates that no particular patient demographic or clinical presentation precludes attempting rapid MR imaging in evaluation of ventricular size or intracranial cyst, because all patients completed an examination without the need for additional imaging to answer the clinical question. Only those with an indication of altered mental status required nominally increased scan durations, which is likely clinically and economically inconsequential.

These results are clinically relevant because the rapid MR imaging technique represents a reasonable means to evaluate hydrocephalus within the pediatric population and can be performed quickly, with nearly the same speed as conventional head CT scanning. It avoids the time and risk associated with conventional pediatric MR imaging scans of the head with the patient under sedation, as well as the ionizing radiation from CT scanning.

Several previous studies relating to pediatric rapid MR imaging have either focused on techniques to optimize shunt catheter tip localization, to describe its various uses, or to estimate potential benefits of radiation dose reduction. ${ }^{9,11-15}$ A recent study demonstrated value in an 8-second gradient-echo technique for determining ventricular size; however, this technique was performed in cases of failed examinations and provides scarce anatomic detail and, subsequently, minimal additional diagnostic information aside from ventricular size. ${ }^{16}$ Despite substantial motion artifacts in some cases, meaningful diagnostic value can be inferred from our results because no cases required conversion to a CT scan or to sedated, standard MR imaging.

We believe that proper selection of appropriate patients for rapid MR imaging examinations can reduce or eliminate the number of CT or sedated MR imaging examinations performed for a number of indications, as long as the referring clinician has a good understanding of its limitations and value. For example, this rapid MR imaging protocol would not be appropriate in pediatric trauma cases or in cases needed to evaluate tumors or infection, given the low sensitivity of rapid MR imaging in the detection of fractures and small amounts of subarachnoid hemorrhage and presumed low specificity in the evaluation of masses and extraaxial fluid collections. As such, these results show how standard CT imaging for indications of hydrocephalus or intracranial cyst has been considerably reduced but remains an essential part of our emergency medicine and oncologic practices. In our institution, these studies are now routinely performed when outpatient, nonemergent imaging of these pediatric patients is needed.

Our study had several limitations, including its retrospective nature. Identifying the causes of imaging failure via subjective chart review can lead to erroneous conclusions regarding the clinical reasoning that brought about a repeated examination. In addition, given the overlap between indications for use of MR imaging and CT scan in the pediatric population, we limited our comparisons to a limited set of hydrocephalus-related indications. Lastly, we did not have long-term follow-up on these patients, and further analysis is needed to determine how rapid MR imaging affects clinical decision-making and outcomes.

AJNR Am J Neuroradiol 34:1836-40 Sep 2013 www.ajnr.org 
Rapid MR imaging examinations being obtained for indications other than hydrocephalus represent an area of potential mismatch between the expectations of the ordering clinicians and the realistic capabilities of this technique. Despite some strengths, rapid MR imaging has a potential limitation in patients with programmable shunts because repeated exposure to magnetic fields may require shunt reprogramming. At our institution, these patients are usually monitored with pre- and post-MR imaging skull radiographs, and as such, the patient's examination risk and exposure to radiation are not zero. In addition, costs to both the patient and the institution should also be factored into decisions to perform rapid MR imaging. We do not recommend eliminating CT scanning in the practice of imaging hydrocephalus, as evening and nighttime MR imaging coverage is not readily available in many centers and timely off-hours imaging is therefore not feasible. In addition, MR imaging carries with it additional safety issues such as projectile injury, which could potentially carry a greater health risk than the dose of radiation from CT, particularly as new low-dose CT techniques are developed.

\section{CONCLUSIONS}

Pediatric rapid brain MR imaging without the need for sedation is an effective and efficient alternative to standard CT imaging for assessment of ventricular size and/or intracranial fluid spaces, irrespective of a child's age or clinical condition. Only patients with altered mental status required nominally increased scan duration, but the time difference was minimal and was without substantial clinical implication. Our practice trends confirm that rapid MR imaging has nearly completely replaced routine, nonemergent CT scanning for these indications.

Disclosures: David Kallmes—UNRELATED: Consultancy: ev3,* Medtronic," Codman; ${ }^{*}$ Comments: Planning and implementation of clinical trials and core laboratory duties; Grants/Grants Pending: ev3,* Sequent Medical,* Cordis,* Codman,* MicroVention; ${ }^{\star}$ Royalties: UVA Patent Foundation, Comments: Spine fusion license; Travel/Accommodations/Meeting Expenses Unrelated to Activities Listed: MicroVention.*

\section{REFERENCES}

1. Coté CJ. Strategies for preventing sedation accidents. Pediatr Ann 2005;34:625-33

2. Coté GA, Hovis RM, Ansstas MA, et al. Incidence of sedation-related complications with propofol use during advanced endoscopic procedures. Clin Gastroenterol Hepatol 2010;8:137-42
3. Cravero JP, Beach ML, Blike GT, et al. The incidence and nature of adverse events during pediatric sedation/anesthesia with propofol for procedures outside the operating room: a report from the Pediatric Sedation Research Consortium. Anesth Analg 2009;108:795-804

4. Brenner D, Elliston C, Hall E, et al. Estimated risks of radiationinduced fatal cancer from pediatric CT. AJR Am J Roentgenol 2001;176:289-96

5. Brenner DJ, Sachs RK. Estimating radiation-induced cancer risks at very low doses: rationale for using a linear no-threshold approach. Radiat Environ Biophys 2006;44:253-56

6. Brenner DJ. Estimating cancer risks from pediatric CT: going from the qualitative to the quantitative. Pediatr Radiol 2002;32:228-23; discussion 242-44

7. Goske MJ, Applegate KE, Boylan J, et al. The Image Gently campaign: working together to change practice. AJR Am J Roentgenol 2008;190:273-74

8. The ALARA (as low as reasonably achievable) concept in pediatric CT intelligent dose reduction. Multidisciplinary conference organized by the Society of Pediatric Radiology. August 18-19, 2001. Pediatr Radiol 2002;32:217-313

9. Koral K, Blackburn T, Bailey AA, et al. Strengthening the argument for rapid brain MR imaging: estimation of reduction in lifetime attributable risk of developing fatal cancer in children with shunted hydrocephalus by instituting a rapid brain MR imaging protocol in lieu of head CT. AJNR Am J Neuroradiol 2012;33:1851-54

10. Forbes KP, Pipe JG, Karis JP, et al. Brain imaging in the unsedated pediatric patient: comparison of periodically rotated overlapping parallel lines with enhanced reconstruction and single-shot fast spin-echo sequences. AJNR Am J Neuroradiol 2003;24:794-98

11. Iskandar BJ, Sansone JM, Medow J, et al. The use of quick-brain magnetic resonance imaging in the evaluation of shunt-treated hydrocephalus. J Neurosurg 2004;101(2 Suppl):147-51

12. Missios S, Quebada PB, Forero JA, et al. Quick-brain magnetic resonance imaging for nonhydrocephalus indications. J Neurosurg Pediatr 2008;2:438-44

13. Penzkofer AK, Pfluger T, Pochmann $Y$, et al. MR imaging of the brain in pediatric patients: diagnostic value of HASTE sequences. AJR Am J Roentgenol 2002;179:509-14

14. Ashley WW Jr, McKinstry RC, Leonard JR, et al. Use of rapid-sequence magnetic resonance imaging for evaluation of hydrocephalus in children. J Neurosurg 2005;103(2 Suppl):124-30

15. Miller JH, Walkiewicz T, Towbin RB, et al. Improved delineation of ventricular shunt catheters using fast steady-state gradient recalled-echo sequences in a rapid brain MR imaging protocol in nonsedated pediatric patients. AJNR Am J Neuroradiol 2010;31: 430-35

16. Wait SD, Lingo R, Boop FA, et al. Eight-second MRI scan for evaluation of shunted hydrocephalus. Childs Nerv Syst 2012;28:1237-41 